\title{
Pathological prognostic factors of retroperitoneal liposarcoma: comprehensive clinicopathological analysis of 124 cases
}

\author{
Pingping Sun ${ }^{1}, \mathrm{Ru} \mathrm{Ma}{ }^{2}$, Gang Liu ${ }^{2}$, Lingling Wang ${ }^{1}$, Hong Chang ${ }^{1}$, Yan $\mathrm{Li}^{1,2}$ \\ ${ }^{1}$ Department of Pathology, Beijing Shijitan Hospital, Capital Medical University, Beijing, China; ${ }^{2}$ Department of Peritoneal Cancer Surgery, Beijing \\ Shijitan Hospital, Capital Medical University, Beijing, China \\ Contributions: (I) Conception and design: P Sun; (II) Administrative support: Y Li; (III) Provision of study materials or patients: G Liu; (IV) \\ Collection and assembly of data: H Chang; (V) Data analysis and interpretation: R Ma, L Wang; (VI) Manuscript writing: All authors; (VII) Final \\ approval of manuscript: All authors. \\ Correspondence to: Yan Li. Departments of Peritoneal Cancer Surgery and Pathology, Beijing Shijitan Hospital, Capital Medical University, No. 10 \\ Tieyi Road, Yangfangdian Street, Haidian District, Beijing 100038, China. Email: liyansd2@mail.ccmu.edu.cn.
}

Background: Retroperitoneal liposarcoma (RLS) is a type of rare malignant tumor. The aim of the present study was to study the correlations between the clinicopathological characteristics and the prognostic factors of RLSso as to delineate independent pathological indicators affecting prognosis.

Methods: The RLS pathological specimens were collected at Beijing Shijitan Hospital Affiliated to Capital Medical University from 2008 to 2018, and were used to study the histopathological features, including tumor size, histological types, lymphatic metastasis, tumor emboli in the blood and lymph vessels, and nerve invasion; immunohistochemical features, including Ki-67 and P53 proteins; and molecular pathological features, such as MDM2 (murine double minute2) gene amplification. Clinical treatment and follow-up data were collected, and the survival rate was calculated by Kaplan-Meier method. Univariate and multivariate survival analyses were performed to study the independent pathological factors.

Results: Of the 124 RLS cases, 63 were males (50.8\%) and 61 were females (49.2\%), with a median age of 52 years (range, 22-80 years). There were 51 cases (41.1\%) of well-differentiated liposarcoma, 73 cases (57.3\%) of dedifferentiated liposarcoma (DDL); 3 cases (2.4\%) with lymph node metastases; 4 cases $(3.2 \%)$ with tumor emboli in the vessels; 21 cases (16.9\%) with $\mathrm{Ki}-67$ label index $<20 \%$ and 103 cases $(83.1 \%)$ with Ki-67 label index $\geq 20 \%$; and 81 cases (65.3\%) with P53 $<60 \%$ and 43 cases $(24.7 \%)$ with P53 $\geq 60 \%$. Fluorescence in situ hybridization studies revealed MDM2 gene amplification. Univariate analysis found the following pathological factors were correlated with prognosis: histopathological classification, recurrence, mitosis, tumor necrosis, Ki-67 label index, and P53. Multivariate analysis by Cox proportional hazard model found the following three independent pathological prognostic factors: age [hazard ratio (HR): 1.798, $\mathrm{P}=0.018$ ], tumor site (HR: 1.263, $\mathrm{P}=0.002)$, tumor necrosis (HR: 1.984, $\mathrm{P}=0.024)$, and recurrence (HR: 2.161, $\mathrm{P}=0.049$ ).

Conclusions: Age, tumor necrosis, tumor site, and recurrence could be independent pathological prognostic factors for RLS.

Keywords: Retroperitoneal liposarcoma (RLS); pathology; immunohistochemistry; prognosis

Submitted Jan 15, 2021. Accepted for publication Mar 30, 2021.

doi: $10.21037 /$ atm-21-972

View this article at: http://dx.doi.org/10.21037/atm-21-972

\section{Introduction}

Soft tissue sarcoma in adult patients accounts for about $1 \%$ of all newly diagnosed tumors, with an incidence rate of 3-6/100,000 (1). Liposarcoma is the most common soft tissue sarcoma, accounting for about $20 \%$ of all malignant mesenchymal tumors (2). Liposarcoma mainly 
occurs in the limb trunk and retroperitoneum, and other rare sites include the mediastinum (3), pleural cavity (4), paratesticular $(5,6)$, spermatic cord (7), esophagus (8), and uterus (9). The clinical site of liposarcoma is retroperitoneal that was known as retroperitoneal liposarcoma.

RLS is a type of rare malignant tumor posing considerable clinical challenges due to its size and deep location. RLS is insensitive to radiotherapy and chemotherapy, and the main treatment is radical resection. Unfortunately, postoperative local recurrence is often frequent and quick, and is a major cause of death for RLS patients. In terms of tumor biology, RLS is highly heterogeneous with several different pathological types (10). The 2020 edition of the World Health Organization classification of soft tissue and bone tumor divides liposarcoma as atypical lipomatous tumor/ well-differentiated liposarcoma (ALT/WDL), myxoid liposarcoma (MLS), pleomorphic liposarcoma (PLPS), and dedifferentiated liposarcoma (DDL) (2). For RLS, ALT/ WDL and DDL are the most common pathological types. MLS rarely occurs in the retroperitoneal space, and has usually the metastasized from other sites. PLPS occurs on the extremities in two thirds of cases.

In the present study, we investigated the clinicopathological features and prognosis of RLS in a large sample of patients receiving uniform treatment at our hospital to explore the prognostic pathological factors.

We present the following article in accordance with the REMARK reporting checklist (available at http://dx.doi. org/10.21037/atm-21-972).

\section{Methods}

\section{Clinical data}

We systematically collected the clinical data and followup information on RPL patients treated at Beijing Shijitan Hospital Affiliated to Capital Medical University from January 2008 to January 2018. The clinical data included age, sex, main symptoms and signs, surgical and medical treatment information, pathological diagnosis, and survival. The overall survival (OS) was calculated from the date of surgery to the last follow-up (October 31, 2020) or the death date. Only patients with complete clinicopathological and follow-up information were included in the study. The pathological specimens of patients who had not received any antitumor therapy before surgery were graded by Fédération nationale des centres de lutte contre le cancer (FNCLCC). The study protocol was approved by the ethics committee of Beijing Shijitan Hospital. All procedures performed in this study involving human participants were in accordance with the Declaration of Helsinki (as revised in 2013). Individual consent for this retrospective analysis was waived.

\section{Routine pathological study and immunobistochemistry (IHC)}

The pathological data included gross pathology, histopathology, and IHC.

For gross pathology, tumor site, size, morphology, and the relationships between tumor and surrounding tissues were recorded.

For histopathological and IHC studies, all specimens were fixed in $10 \%$ buffered neutral formalin and sliced into 3 - $\mu$ m-thick serial sections. IHC staining was performed on 3- $\mu \mathrm{m}$ sections of formalin-fixed, paraffin-embedded tissue. Slides were deparaffinized, rehydrated in a graded series of alcohol, and microwave-treated for $10 \mathrm{~min}$ in citrate buffer ( $\mathrm{pH}$ 6.0). Endogenous peroxidase activity was quenched using $0.3 \%$ hydrogen peroxide. The specimens were processed in an automatic IHC staining machine with standard protocols (BIOCARE MEDICAL, LLC, intelliPATH FLX, Boston, the United States; DAKO Real EnVision Detection System, Basel, Switzerland). Histopathological features included tumor type, tumor gross pattern, tumor invasion into the peripheral tissues, nerve invasion, lymph node metastasis, and vascular tumor embolus.

IHC antibodies included Ki-67 (OriGene, Clone UMAB107) and P53 (OriGene, clone DO7) and CDK4 (cyclin-dependent kinase 4) (ZSGB Bio Co., Ltd., Clone EP180) and MDM2 (OriGene, Clone 1E6\&17B3) and P16 (ZSGB Bio Co., Ltd., Clone G175-405). All antibodies were incubated at room temperature for $1 \mathrm{~h}$. Diaminobenzidine was used for color development, and Mayer hematoxylin was used for contrast staining. A positive control was established according to the instructions, and phosphatebuffered solution replaced the primary antibody as the negative control. Positive standard was defined as the presence of brownish-yellow or tan particles in the nucleus without cytoplasm staining, as indicated by the positive index of $\mathrm{Ki}-67$, which divided into the $\mathrm{Ki}-67<20 \%$ and Ki-67 $\geq 20 \%$ categories. P53 was defined as positive when there were brownish or tan particles in the nucleus, but the cytoplasm was not stained, and was divided into P53 $<60 \%$, P $53 \geq 60 \%$, or absent. P16 and CDK4 was defined 
as positive when there were brownish or tan particles in the nucleus and cytoplasm, MDM2 was defined as positive when there were brownish or tan particles in the nucleus. The slides were reviewed by two senior pathologists who were blinded to the clinical data and follow-up status.

\section{Fluorescence in situ bybridization (FISH)}

The MDM2 mutation status was assessed with FISH molecular detection. The conventional tissue sections were dewaxed-dehydrated, pretreated, denatured with the MDM2 probe (Embapine, Guangzhou, China), washed successively with $2 \times$ sodium citrate buffer and gradient ethanol, 4',6-diaminyl 2-phenyl indoles (DAPI), and sealed with a neutral resin. Using a Zeiss AX10 fluorescence microscope, 200 intracellular fluorescence signals were counted under DAPI (diamidino-phenyl-indole) (Embapine, Guangzhou, China), FITC (Fluorescein Isothiocyanate) (Embapine, Guangzhou, China), and RHOD (rhodamine red) filter oil, and no overlapping cells were counted. The interpretation criteria were 4R2G and GSPMDM2/CSP12 $\geq 2.0$, indicating $M D M 2$ gene amplification.

\section{Statistical analysis}

The database was established with Microsoft Excel 2019 and analyzed with SPSS version 21.0 (IBM, Armonk, NY, USA). Independent sample $t$-test was used to test the measurement data in accordance with normal distribution, and nonparameter test was used to analyze the measurement data in non-normal distribution. Statistical data were obtained by $\chi^{2}$-test and Fisher's exact probability method. The cutoff value of the receiver-operating characteristic curve (ROC) was set for age, recurrence frequency, tumor size, Ki-67-positive index, and P53-positive percentage. Survival analysis was determined by Kaplan-Meier method and logrank test, and Cox regression model was used to analyze the independent prognostic factors. Two-sided $\mathrm{P}<0.05$ was considered statistically significant.

\section{Results}

\section{Clinical features}

A total of 124 RLS patients were included, including 63 males (50.8\%) and 61 females (49.2\%). The median age was 52 years (22-80 years). All patients received surgical treatment, including 68 patients $(57.9 \%)$ who underwent surgical resection alone and 56 patients $(36.8 \%)$ who underwent surgical resection plus intraperitoneal perfusion chemotherapy, or surgery combined with radiotherapy. The typical CT findings were a mass of soft tissue density in the retroperitoneal space, internal with fat density components. In DDL, the density was uneven, and obvious enhancement components and low density area of necrosis and hemorrhage were found (Figure 1). Detailed clinicopathological features are listed in Table 1.

\section{Histopathological characteristics}

Macroscopy of the tumor consists usually of a large, wellcircumscribed, lobulated mass. The cut surface of the tumor shows yellow-tan-gray areas, often mixed with necrosis and hemorrhage. Histopathologically, 51 cases (41.1\%) were diagnosed with ALT/WDL and 66 (57.3\%) with DDL. ALT/WDL can be subdivided morphologically into three subtypes: adipocytic (lipoma-like), sclerosing, and inflammatory. ALT is composed of mature adipocyte proliferation in which there are significant variations in cell size (Figure 2A). Numerous hyperchromatic stromal cells were present within the fibrous septa, and a varying number of monovacuolated or multivacuolated lipoblasts could be observed (Figure 2B). The subtype of sclerosing liposarcoma is composed of dense, collagenous fibrosis regions, with certain atypia of spindle cells and a small number of vesicular adipoblasts. Inflammatory liposarcoma subtypes contain lymphocytes and plasma cells in varying amounts in ALT and sclerosing lipomas, and may form nodular aggregation foci or germinal centers (Figure 2C).

DDL usually consists of large, multinodular, yellow masses containing discrete, solid, often tan-gray, nonlipomatous (dedifferentiated) areas. The transition between the lipomatous and the dedifferentiated areas may be gradual or abrupt. The histological DDL shows a spectrum of morphological appearances, although the majority show features of UPS (undifferentiated pleomorphic sarcoma) or spindle-cell sarcoma, with high-to-moderate cellularity, pleomorphism, and moderate-to-marked cellular atypia with cells disposed in loose fascicles, patternless distributions, or sometimes with a storiform "MFH (malignant fibrous histiocytoma)-like" architecture, within variably fibrous stroma. The mitotic index is variable; it can be high, but often is lower than usually observed in other UPS. Variable amounts of necrosis can be seen. A minority of DDL, however, are of low cellularity and comprise only histologically low-grade areas resembling fibromatosis, or low-grade fibromyxoid sarcoma; these are composed 

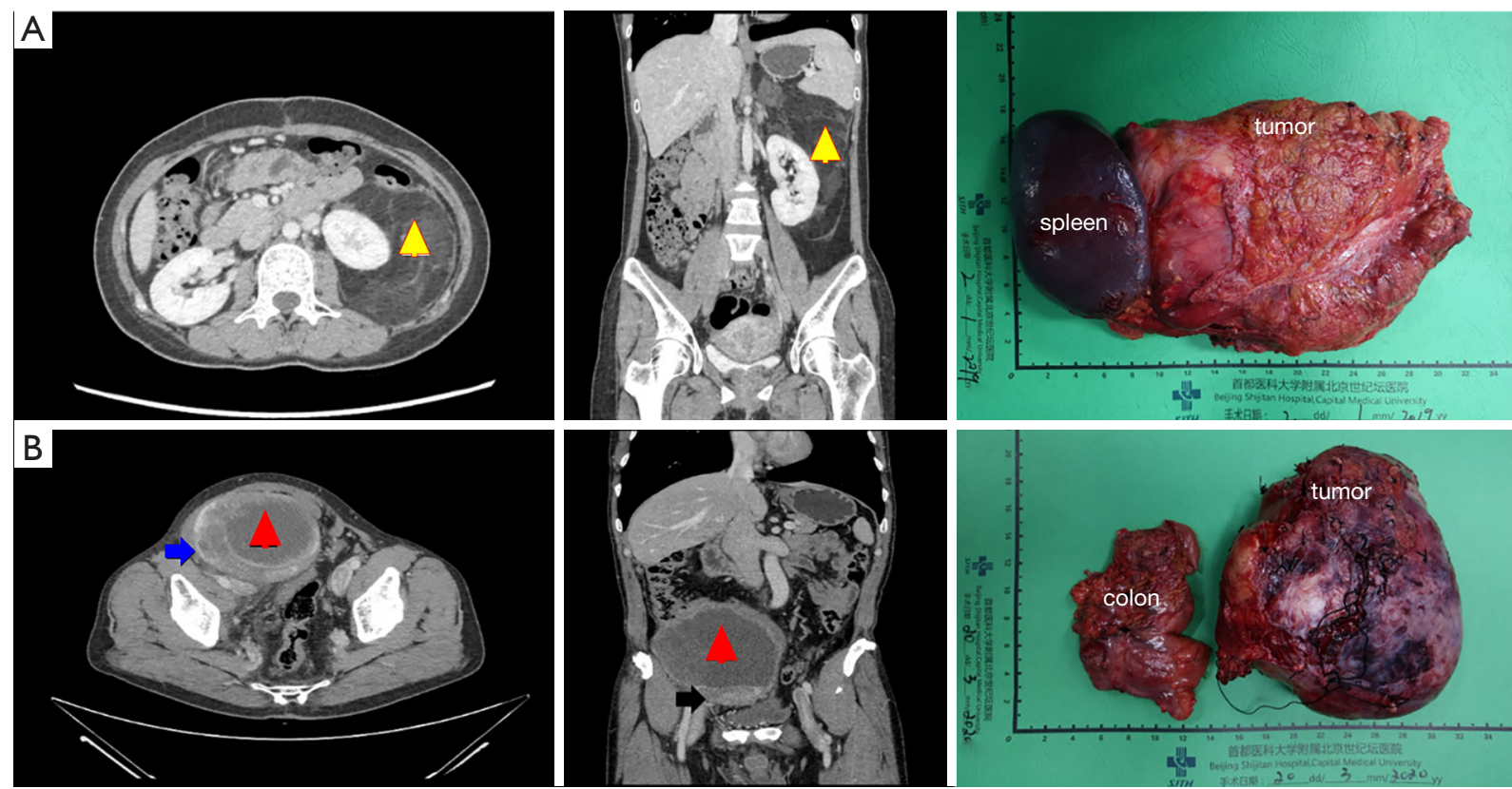

Figure 1 Typical performance in computed tomography images and gross anatomy. (A) Atypical lipomatous tumor/well-differentiated liposarcoma. Yellow triangle shows the tumor. (B) Dedifferentiated liposarcoma. Red triangle shows the cystic necrosis and hemorrhage, Blue arrow shows the solid portion of the tumor.

of sparse-to-moderate cellular proliferations comprising loose fascicles or pattern-less distributions of fibroblastlike spindle cells with mild nuclear atypia and low mitotic activity.

DDL varies according to its pattern of high- or lowgrade dedifferentiation. Morphologically low grade DDL presented invasive fibromatosis, myxofibrosarcoma, or inflammatory myofibroblastoma, benign nerve sheath neoplasm or low-grade fibromyxoid sarcoma; inflammatory DDL can resemble IgG4-associated sclerosing lesions or inflammatory myofibroblastic tumor. High grade heterogenic components may occur, such as fibrosarcoma (Figure 2D), rhabdomyosarcomas, leiomyosarcomas, UPS and angiosarcomas, osteosarcomas (Figure 2E). In some cases, meningeal epithelioid swirls are seen. Some patterns of DDL closely resemble pleomorphic liposarcoma when their dedifferentiated component is an aliphatic sarcoma (Figure $2 F$ ), and DDL often present a background of mucinous degeneration. Highly differentiated liposarcoma with metastasis in the lymph nodes, vascular tumor thrombus, and nerve invasion were observed (Figure 2G,H,I).

Via IHC, 81 cases $(65.3 \%)$ had a P53-positive percentage $<60 \%$, and 43 cases (24.7\%) had P53 $\geq 60 \%$. In 21 cases, the $\mathrm{Ki}-67$-positive index was $<20 \%(16.9 \%)$ and $\geq 20 \%$ in 103 cases $(22.8 \%)$. All available cases underwent immunohistochemistry for both P16 (Figure 27), MDM2 and CDK4 (Figure 2K).

In terms of molecular pathology, when it was difficult to differentiate DDL from MLS and PLPS. FISH assay was used to detect the increased red signals in liposarcoma tumor cells, indicating the amplification of the MDM2 gene (Figure 2L).

\section{Follow-up and survival}

The median follow-up time of 124 patients was 76.4 months [95\% confidence interval (CI): 60.7-92.1 months] and the median OS was 27.4 months (95\% CI: 13.7-41.0 months). Eighty patients died (61.4\%), and 44 patients survived (38.6\%).The accumulative 1-, 3-, and 5-year survival rates were $90.3 \%, 71 \%$, and $50.2 \%$, respectively (Figure $3 A$ ).

\section{Univariate and multivariate analyses of survival-related factors}

Based on the ROC curve analysis, we determined that the cut-off value for age was 56 years, 3/10 high power field (HPF) for pathological mitosis, $24.5 \mathrm{~cm}$ for tumor size, 
Table 1 Major clinic-pathological features of 124 RLS cases in this study

\begin{tabular}{|c|c|}
\hline Characteristics & $\mathrm{n}(\%)$ \\
\hline \multicolumn{2}{|l|}{ Sex } \\
\hline Male & $63(50.8)$ \\
\hline Female & $61(49.2)$ \\
\hline \multicolumn{2}{|l|}{ Age (years) } \\
\hline Median & 52 \\
\hline Range & $22-80$ \\
\hline \multicolumn{2}{|l|}{ Tumor location } \\
\hline Left retroperitoneal space & $55(44.4)$ \\
\hline Right retroperitoneal space & $30(24.2)$ \\
\hline Pelvis & $3(2.4)$ \\
\hline Whole abdomen & $36(29.0)$ \\
\hline \multicolumn{2}{|l|}{ Treatment } \\
\hline Surgery alone & $68(54.8)$ \\
\hline Surgery + HIPEC & $48(38.7)$ \\
\hline Surgery + chemotherapy + radiotherapy & $8(6.5)$ \\
\hline \multicolumn{2}{|l|}{ Detailed information on surgery } \\
\hline Tumor resection alone & $35(28.2)$ \\
\hline Tumor resection + adjacent organs & $89(71.8)$ \\
\hline \multicolumn{2}{|l|}{ Pathological type } \\
\hline ALT/WDL & $51(41.1)$ \\
\hline DDL & $73(57.3)$ \\
\hline
\end{tabular}

ALT/WDL, atypical lipomatous tumor/well-differentiated liposarcoma; DDL, dedifferentiated liposarcoma; HIPEC, hyperthermic intraperitoneal chemotherapy; NA, not applicable; RLS, retroperitoneal liposarcoma.

$20 \%$ for Ki-67 index, and $60 \%$ for P53-positive percentage. Univariate survival analysis showed that the following clinicopathological factors were correlated with OS: tumor site, histological type, nerve division, necrosis, recurrence, and P53-positive percentage (Table 2). The median OS was longer in the left retroperitoneal space, right retroperitoneal space, and pelvis compared with the whole abdomen $(38.8,27.4,56.3$ vs. 18.4 months, $\mathrm{P}=0.013)$. The median OS was longer in cases without recurrence than those with recurrence (102.2 vs. 21.1 months, $\mathrm{P}<0.001)$. The median OS was longer in cases with WDL than those with DDL (65.9 vs. 19.5 months, $\mathrm{P}<0.001$ ), in cases with pathological mitosis $3 / 10 \mathrm{HPF}$ than those $\geq 3 / 10 \mathrm{HPF}$ (48.1 vs. 11.1 months, $\mathrm{P}<0.001)$, in cases without tumor necrosis than those with tumor necrosis (69.1 vs. 19.1 months, $\mathrm{P}<0.001)$, in cases without tumor ossification than those with ossification $(\mathrm{P}=0.020)$, and in cases with $\mathrm{P} 53$ positive percentage $<60 \%$ than those $\geq 60 \%$ (32.4 vs. 24.8 , $\mathrm{P}=0.024)$.

Multivariate analysis of the variables that were statistically significant according to the univariate analysis showed that age [hazard ratio (HR): $1.798, \mathrm{P}=0.018$ ], tumor site (HR: 1.263, $\mathrm{P}=0.002$ ), tumor necrosis (HR: 1.984, $\mathrm{P}=0.024$ ), and recurrence (HR: 2.161, $\mathrm{P}=0.049$ ) were 4 independent clinicopathological indicators for OS in these patients (Table 3 and Figure 3B,C,D).

\section{Discussion}

In the present study, we summarized our experience in treating soft tissue liposarcoma at our center. RLS accounted for $36 \%$ of all malignant mesenchymal tumors at our hospital from 2008 to 2018. The average age of occurrence was 50-60 years, with an equal male:female distribution. The 1-, 3-, and 5-year OS rates were 90.3\% (112/124), 71\% (88/124), and 50.2\% (72/124), respectively.

In gross pathology, RLS in this study usually consists of a large, Well-circumscribed, lobulated mass. Variable consistencies are present, from firm grey to gelatinous areas. Some studies also reported on tumor sections with multilocular cystic features (11). The maximum tumor size in our cases was about $45 \mathrm{~cm}$ in diameter. The maximum diameter of the tumor was slightly larger than that reported in the literature (12). After surgical resection, the local recurrence rate was about $78.9 \%$ and tumor-specific mortality rate was about $61.4 \%$, both of which were higher than those reported in the literature (10). Compared with other types of pleomorphic sarcomas, DDL still has a relatively good prognosis (13).

Differential diagnoses between RLS and other soft tissue tumors are sometimes problematic. WDL should be differentiated from pleomorphic lipoma and brown tumor, and DDL should be differentiated from gastrointestinal stromal tumor, single-phase spindle cell synovial sarcoma, pleomorphic undifferentiated sarcoma, malignant peripheral schwannoma, fibrosarcoma, leiomyosarcoma, and rhabdomyosarcoma. The co-expression of MDM2 and CDK4 by IHC staining important in the diagnosis of DDL due to the presence of consistent amplification and overexpression in the 12q14-15 region of the liposarcoma, and the high consistency between the strong expression 

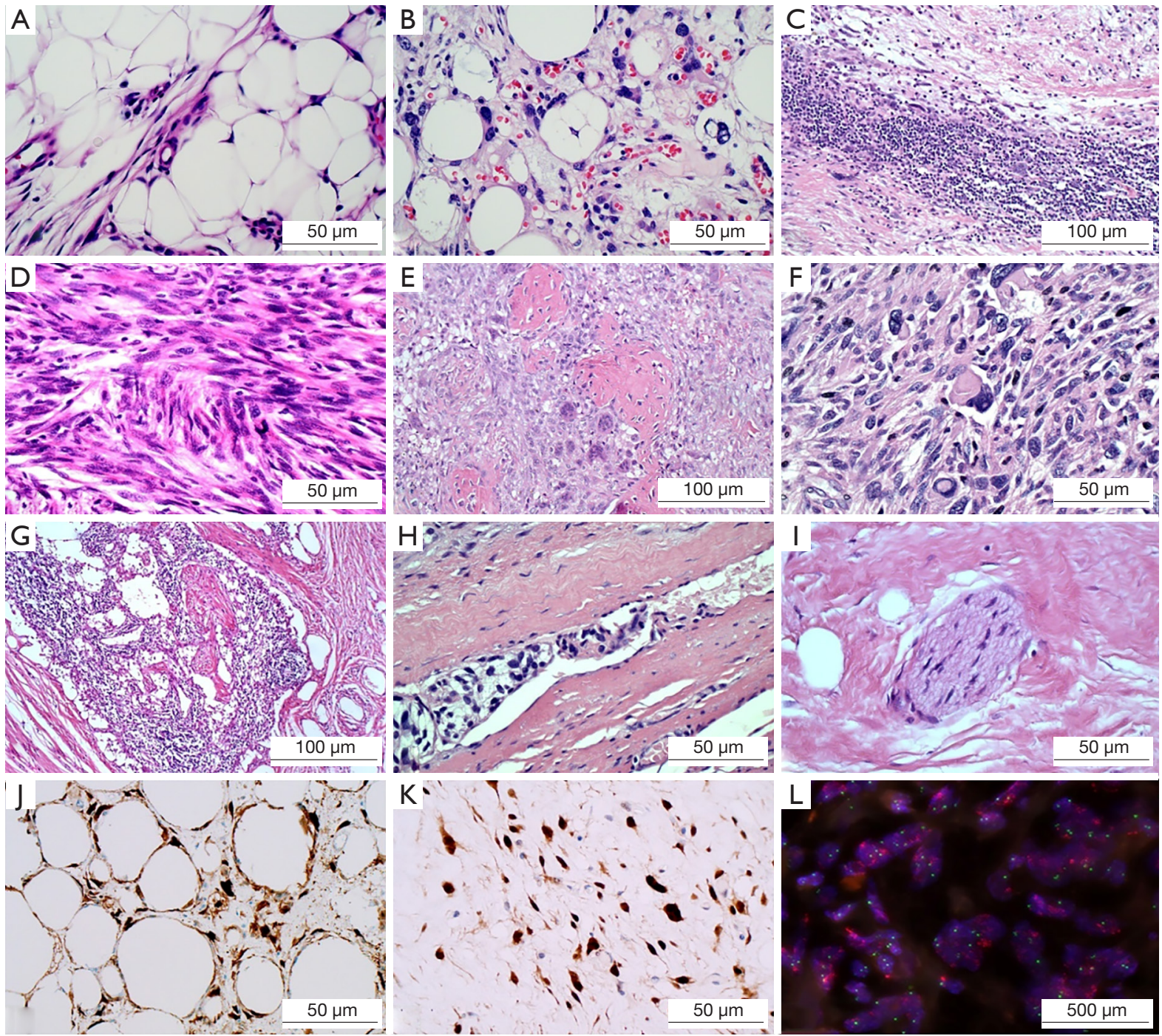

Figure 2 Typical histopathological features of 124 RLS patients in the study. (A) Well-differentiated liposarcoma fat vacuoles vary in size [hematoxylin-eosin (HE), 400x]; (B) A variable number of lipoblasts can be seen in WDL (HE 400x); (C) Abundant lymphocytes and scattered bizarre stromal cells are present in the field of inflammatory subtype of WDL (HE 200x). (D) Dedifferentiated component was fibrosarcoma (HE 400×). (E) Dedifferentiated liposarcoma may feature a distinctive osteosarcomatous area or osteogenic differentiation (HE 400x). (F) This field shows PLPS -like morphology that feature pleomorphic tumour cell with bizarre cell nucleus (HE 400x). (G) Sclerosing liposarcoma in the lymph nodes (HE 200x). (H) Tumor emboli can be seen in the vascular (HE 400x). (I) Liposarcoma cells invading the nerve (HE 400×). (J) Diffuse nuclear and cytoplasm expression of P16 is consistently observed in dedifferentiated liposarcoma (EnVision method 400x). (K) Diffuse nuclear expression of CDK4 is consistently observed in dedifferentiated liposarcoma (EnVision method 400x). (L) MDM2 gene amplification (fluorescence in-situ hybridization method 1,000x).

of MDM2 and CDK4 protein and corresponding gene amplification. The sensitivity of P16 is high, but the specificity is low. Therefore, the combination of 3 antibodies is important for the diagnosis of liposarcoma. It's very difficult to distinguish between DDL or WDL with mucus degeneration and MLS. especially to DDL mucous degeneration serious when, often misdiagnosed as MLS, should have more materials, carefully read for a slime variable area with dense areas migration transition is shown in, usually a MLS mainly occurs on the trunk and 

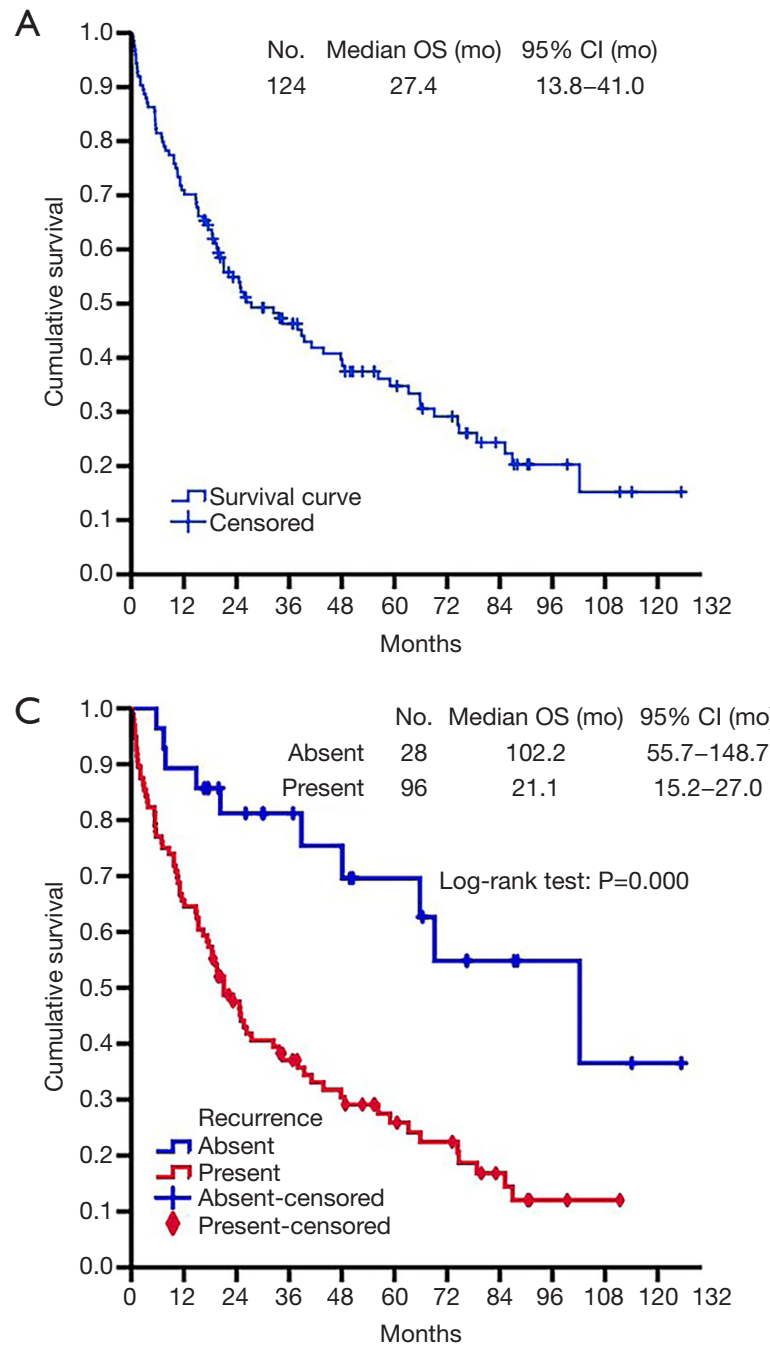
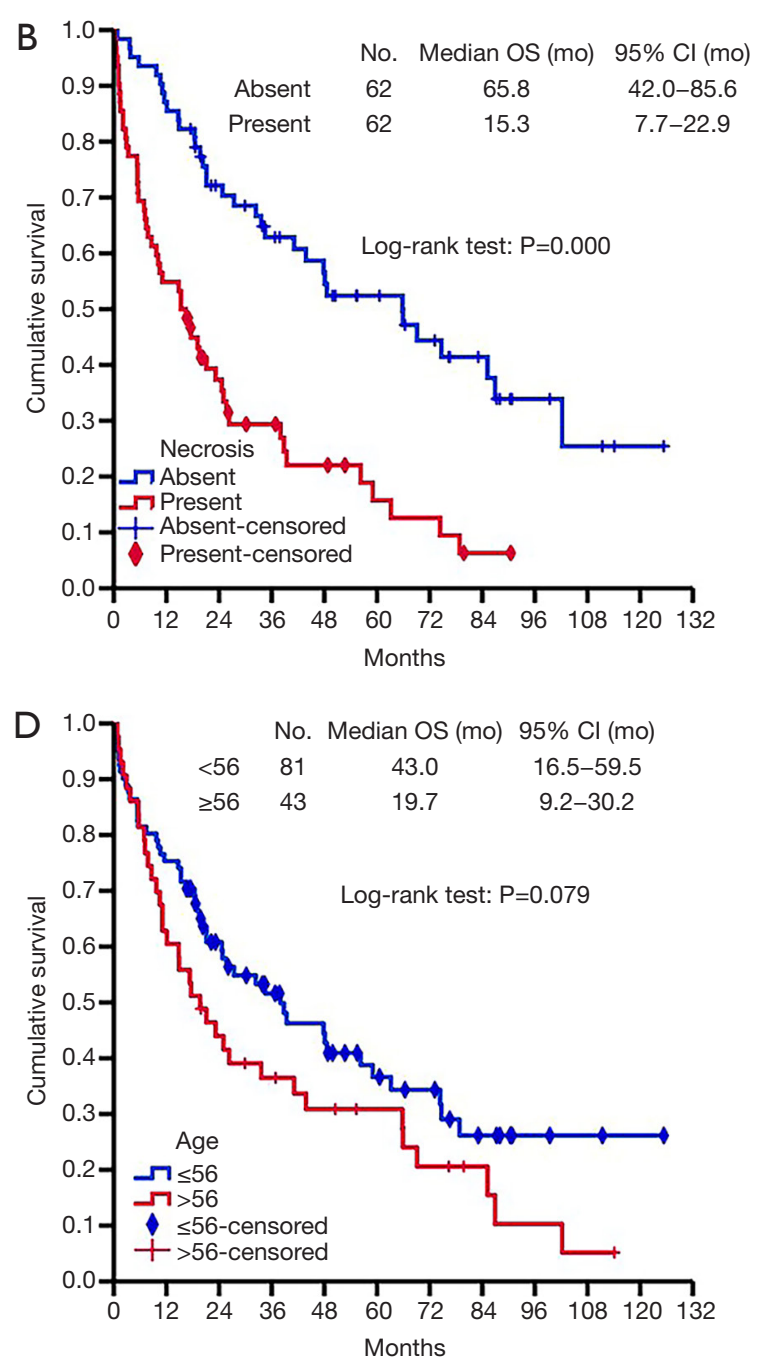

Figure 3 Survival curve. (A) Overall survival curve of 124 liposarcoma patients; (B) liposarcoma necrosis; (C) liposarcoma recurrence; (D) age at diagnosis.

limbs, rarely occurs in retroperitoneal, IHC NY-ESO-1 to identify them (14). If it is indeed difficult to distinguish, the metastasis from limb and trunk sources should be excluded by detailed medical history, FISH detection is performed using the fusion gene of FUS-DDIT3 and the MDM2 amplification gene, and the fusion gene of FUS-DDIT3 appears in MLS (15), while the MDM2 gene of DDL is amplified. When it was difficult to differentiate DDL from PLPS. PLPS most frequently arises on the extremities or trunk of a slightly older adult population with median age in the seventh decade. Genetically, pleomorphic liposarcoma is not associated with the 12q13-15 amplicon and does not show MDM2 amplification with FISH, and CDK4 and MDM2 coexpression is present in DDL but not PLPS.
These can be difficult to distinguish from sclerosing WDL or from cellular versions of WDL. Histologically, low-grade DDL have shown behavior resembling traditional DDL (with the capacity to metastasize), rather than of WDL. In the study of 155 DDL by Henricks et al., neither low-grade dedifferentiation nor a low percentage of dedifferentiation was associated with any improvement in outcome (16).

Prominent myxoid stroma can be a feature of both DDL and WDL, particularly within the retroperitoneum, abdominal cavity, and spermatic cord. DDL with myxoid stroma can show a prominent coarse plexiform vascular pattern similar to that seen in myxofibrosarcoma, and sometimes a pulmonary edema-like pattern similar to that of MLS (17). Usually the myxoid areas contain atypical 
Table 2 Univariate analysis of overall survival in 124 RLS cases

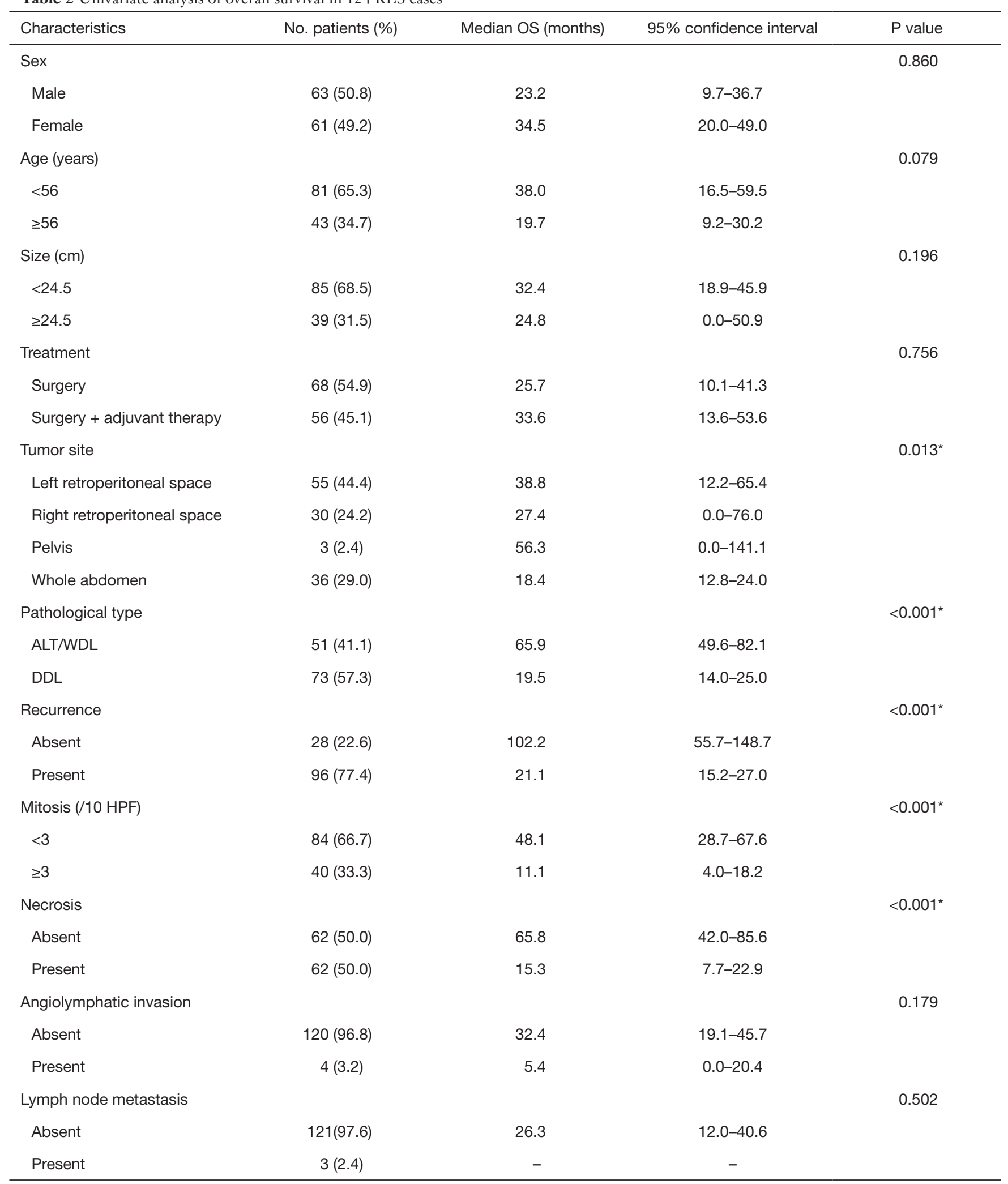

Table 2 (continued) 
Table 2 (continued)

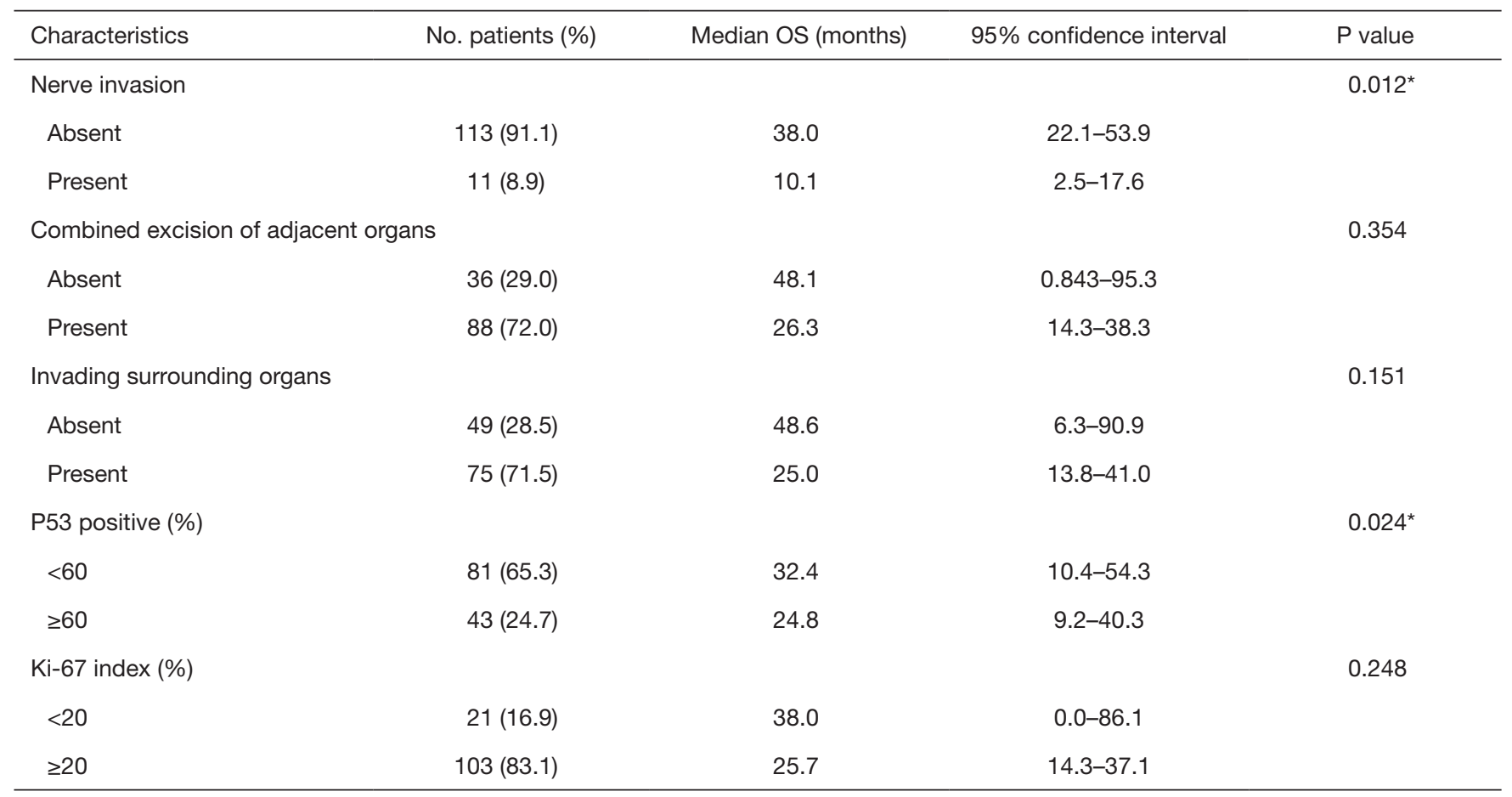

*, $\mathrm{P}<0.05$ indicates statistical significance. ALT/WDL, atypical lipomatous tumor/well-differentiated liposarcoma; DDL, dedifferentiated liposarcoma; HPF, high power field; OS, overall survival.

Table 3 Multivariate analysis of independent factors influencing overall survival

\begin{tabular}{lccc}
\hline Characteristics & Hazard ratio & 95\% confidence interval & P value \\
\hline Sex (male vs. female) & 1.145 & $0.724-1.812$ & 0.562 \\
Age (<56 vs. $\geq 56$ years) & 1.972 & $1.202-3.235$ & $0.007^{*}$ \\
Tumor site & 1.263 & $1.062-1.504$ & $0.002^{*}$ \\
Pathological type (ALT/WDL vs. DDL) & 1.039 & $0.833-1.295$ & 0.736 \\
Mitosis (<3/10 HPF vs. $\geq 3 / 10$ HPF) & 1.424 & $0.843-2.403$ & 0.186 \\
Necrosis (yes vs. no) & 1.997 & $1.172-3.402$ & $0.011^{*}$ \\
Nerve invasion (yes vs. no) & 1.541 & $0.714-3.325$ & 0.270 \\
Recurrence (yes vs. no) & 3.067 & $1.516-6.205$ & $0.002^{*}$ \\
Ossification (yes vs. no) & 1.465 & $0.715-3.000$ & 0.297 \\
P53 (<60\% vs. $\geq 60 \%)$ & 1.424 & $0.837-2.424$ & 0.192 \\
\hline
\end{tabular}

*, $\mathrm{P}<0.05$ indicates statistical significance. ALT/WDL, atypical lipomatous tumor/well-differentiated liposarcoma; DDL, dedifferentiated liposarcoma; HPF, high power field.

spindle or ovoid cells with a myxofibrosarcoma-like morphology, but more rarely these can largely lack atypia and resemble MLS. Cytogenetic and molecular genetic studies have shown that MLS-like areas in RLS represent part of the morphological spectrum of WDL/DDL, rather than true MLS. Other features that have been noted include rare DDL with inflammatory MFH-like morphology, with large, atypical histiocyte-like cells within a prominent 
inflammatory background, including eosinophils, neutrophils, lymphocytes, and foamy histiocytes, or features resembling inflammatory myofibroblastic tumor, meningothelial-like whorls, and metaplastic bone formation, which are also rare features.

Multifactor analysis showed that age, tumor site, tumor necrosis, and recurrence were the most important independent prognostic factors of OS. Median OS was significantly longer at $<56$ years than at $\geq 56$ years ( $38 v s .19 .7$ months). Although tumor size was not an independent prognostic factor, the median survival was significantly longer at $<25.5 \mathrm{~cm}$ than at $\geq 25.5 \mathrm{~cm}$ (43.8 vs. 24.8 months). RLS located in 1 side of the abdomen or pelvis has a better prognosis than liposarcoma located in the whole abdomen. Therefore, these features are still clinically important.

Tumor histological and molecular features have important impacts on clinical outcomes. The statistical results showed that liposarcoma with a high expression of $\mathrm{Ki}-67$ was more likely to relapse than that with a low expression. Gronchi et al. reported that FNCLCC grade had a significant predictive effect on the prognosis of RLS (18). In this study, single-factor prognostic analysis showed that pathological type, mitosis, and necrosis were significantly correlated with prognosis, but multivariate prognostic analysis showed that only necrosis has statistical significance. Lymphatic vascular invasion seems to be an important adverse pathological factor in soft tissue tumors of the trunk and limbs. Even when other established prognostic factors are taken into account, lymphatic vessel invasion can predict worse survival (19). In this study, but the embolus was not significantly correlated with OS.

Although multifactor analysis showed that age, tumor site, tumor necrosis, and recurrence were the most important prognostic factors, tumor size, mitotic, vascular tumor embolus, nerve invasion, and other indicators should also be considered in treatment decisions and may be helpful in stratifying patients in clinical trials. There was no statistically significant difference in survival between surgical treatment alone and additional thermal perfusion therapy or chemoradiotherapy, which was consistent with other retrospective studies in the literature $(10,13,20,21)$. Therefore, surgical treatment is the most effective way to treat RLS, and the impacts of other treatment options warrant further research.

In conclusion, this study on 124 RLS patients from a single center reveals 4 independent clinicopathological factors affecting OS: age, tumor site, tumor necrosis, and recurrence. Further multicenter studies with a prospective design should be conducted to validate these results and to evaluate the importance of other histopathological and molecular features. The poor prognosis of liposarcoma is a concomitant result of many factors.

\section{Acknowledgments}

Funding: This study was funded by the National Natural Science Foundation of China (No. 82073376), Beijing Municipal Administration of Hospitals' Ascent Plan (DFL20180701), and Beijing Municipal Grant for Medical Talents Group on Peritoneal Surface Oncology (No. 2017400003235J007).

\section{Footnote}

Reporting Checklist: The authors have completed the REMARK reporting checklist. Available at http://dx.doi. org/10.21037/atm-21-972

Data Sharing Statement: Available at http://dx.doi. org/10.21037/atm-21-972

Conflicts of Interest: All authors have completed the ICMJE uniform disclosure form (available at http://dx.doi. org/10.21037/atm-21-972). The authors have no conflicts of interest to declare.

Ethical Statement: The authors are accountable for all aspects of the work in ensuring that questions related to the accuracy or integrity of any part of the work are appropriately investigated and resolved. This study was approved by the institutional review board of ethics committee at Beijing Shijitan Hospital. All procedures performed in this study involving human participants were in accordance with the Declaration of Helsinki (as revised in 2013). Individual consent for this retrospective analysis was waived.

Open Access Statement: This is an Open Access article distributed in accordance with the Creative Commons Attribution-NonCommercial-NoDerivs 4.0 International License (CC BY-NC-ND 4.0), which permits the noncommercial replication and distribution of the article with the strict proviso that no changes or edits are made and the original work is properly cited (including links to both the formal publication through the relevant DOI and the license). See: https://creativecommons.org/ 
licenses/by-nc-nd/4.0/.

\section{References}

1. Muratori F, Frenos F, Bettini L, et al. Liposarcoma: Clinico-pathological analysis, prognostic factors and survival in a series of 307 patients treated at a single institution. J Orthop Sci 2018;23:1038-44.

2. Ikoma N, Feig BW. ASO Author Reflections: Recurrent Retroperitoneal Well-Differentiated Liposarcoma. Ann Surg Oncol 2019;26:543-4.

3. Yang YS, Bai CY, Li ZC, et al. Giant primary liposarcoma of the anterior mediastinum: A case report. Medicine (Baltimore) 2018;97:e12873.

4. Pusiol T, Piscioli I, Rondoni V, et al. Intrathoracic liposarcoma: Case report with emphasis to histogenesis and site of origin classification problems. Lung India 2018;35:186-7.

5. Thomas KL, Gonzalez RJ, Henderson-Jackson E, et al. Paratesticular Liposarcoma Masquerading as an Inguinal Hernia. Urology 2018;113:e5-e6.

6. Mouden K, Wakrim S, Semmar A. Paratesticular liposarcoma: A case report. Pan Afr Med J 2019;33:282.

7. Horitani H, Takezawa K, Fukuhara S, et al. A Case of Spermatic Cord Liposarcoma Difficult to Distinguish from Inguinal Hernia. Hinyokika Kiyo 2019;65:389-92.

8. Liu TT, Wu P, Zhang SD. A case of giant primary liposarcoma of esophagus. Zhonghua Zhong Liu Za Zhi 2018;40:62-3.

9. Kiuchi K, Hasegawa K, Ochiai S, et al. Liposarcoma of the uterine corpus: A case report and literature review. Gynecol Oncol Rep 2018;26:78-81.

10. Zhao M, Xu MX, Wang YB, et al. Updates on histologic diagnosis and differential diagnosis of dedifferentiated liposarcoma. Zhonghua Bing Li Xue Za Zhi 2019;48:573-9.

11. Komine C, Fukuchi M, Sakurai S, et al. Retroperitoneal Liposarcoma with Multilocular Cysts. Case Rep Gastroenterol 2019;13:514-20.

12. Moyon FX, Moyon MA, Tufino JF, et al. Massive retroperitoneal dedifferentiated liposarcoma in a young patient. J Surg Case Rep 2018;2018:rjy272.

Cite this article as: Sun P, Ma R, Liu G, Wang L, Chang H, Li Y. Pathological prognostic factors of retroperitoneal liposarcoma: comprehensive clinicopathological analysis of 124 cases. Ann Transl Med 2021;9(7):574. doi: 10.21037/atm-21-972
13. Mastrangelo G, Coindre JM, Ducimetiere F, et al. Incidence of soft tissue sarcoma and beyond: a populationbased prospective study in 3 European regions. Cancer 2012;118:5339-48.

14. Thomas R, Al-Khadairi G, Roelands J, et al. NY-ESO-1 Based Immunotherapy of Cancer: Current Perspectives. Front Immunol 2018;9:947.

15. Mantilla JG, Ricciotti RW, Chen EY, et al. Amplification of DNA damage-inducible transcript 3 (DDIT3) is associated with myxoid liposarcoma-like morphology and homologous lipoblastic differentiation in dedifferentiated liposarcoma. Mod Pathol 2019;32:585-92.

16. Henricks WH, Chu YC, Goldblum JR, et al. Dedifferentiated liposarcoma: A clinicopathological analysis of 155 cases with a proposal for an expanded definition of dedifferentiation. Am J Surg Pathol 1997;21:271-81.

17. Sioletic S, Dal Cin P, Fletcher CD, et al. Welldifferentiated and dedifferentiated liposarcomas with prominent myxoid stroma: analysis of 56 cases. Histopathology 2013;62:287-93.

18. Gronchi A, Collini P, Miceli R, et al. Myogenic differentiation and histologic grading are major prognostic determinants in retroperitoneal liposarcoma. Am J Surg Pathol 2015;39:383-93.

19. Ethun CG, Lopez-Aguiar AG, Switchenko JM, et al. The Prognostic Value of Lymphovascular Invasion in Truncal and Extremity Soft Tissue Sarcomas: An Analysis from the National Cancer Database. Ann Surg Oncol 2019;26:4723-9.

20. Chouliaras K, Senehi R, Ethun CG, et al. Role of radiation therapy for retroperitoneal sarcomas: An eight-institution study from the US Sarcoma Collaborative. J Surg Oncol 2019;120:1227-34.

21. Ikoma N, Roland CL, Torres KE, et al. Concomitant organ resection does not improve outcomes in primary retroperitoneal well-differentiated liposarcoma: A retrospective cohort study at a major sarcoma center. J Surg Oncol 2018;117:1188-94.

(English Language Editor: R. Scott) 